\title{
Inspiring Technologies for Digital Inclusivity
}

\author{
Kang Leng Chiew \\ Faculty of Computer Science and Information Technology, Universiti Malaysia Sarawak, Kota Samarahan, Malaysia \\ Corresponding author: Kang Leng Chiew (klchiew@unimas.my)
}

\begin{abstract}
This editorial aims to summarise the special issue entitled Inspiring Technologies for Digital Inclusivity, which was prepared from selected papers from the $12^{\text {th }}$ International Conference on IT in Asia (CITA'21). The special issue consists of five scientific articles.
\end{abstract}

\section{Keywords}

Digital inclusivity; E-commerce; COVID-19; Machine learning; Online education.

Digitization has never been more important. Many countries have already channelled their efforts to embrace digital inclusion as their main national agenda. The way forward is to inspire technologies for digital inclusivity. It is necessary for all societies, including disadvantaged groups, to have access to and use of telecommunication and information technology. Efforts to eliminate barriers to access technology are highly required. Organic participation and adaptability of societies in new technology is important to improve and enrich the quality life of individuals in all societies.

In line with this, the theme "Inspiring Technologies for Digital Inclusivity" has been chosen for the $12^{\text {th }}$ International Conference on IT in Asia (CITA'21). CITA'21 is a regular series of biennial conferences organised since 1999 by the Faculty of Computer Science and Information Technology, Universiti Malaysia Sarawak, Malaysia. High quality articles are selected from the conference after a preliminary screening. Nineteen articles were submitted to Acta Informatica Pragensia (AIP) for a series of rigorous reviews. The articles were doubleblind reviewed by at least two international experts in the field. Reviewers were selected by the Special Issue Editor and Section Editor-in-Chief who served as Academic Editor for all the articles. With the recommendation from the reviewers, the Section Editor-in-Chief sent the final notifications of acceptance or rejection to the authors. After further selection and the peer review process, five articles were accepted (acceptance rate $26 \%$ ) for publication in the AIP journal (volume 10, issue 2). 
It is our highest privilege to collaborate with the AIP and publish the selected articles in the special issue. It is noteworthy to mention that the AIP only accepts high quality articles and practises a stringent but effective review process. Furthermore, the voluntary work given to editing and proofreading for the camera-ready articles is exceptional.

This special issue contains five articles that include the area of e-commerce readiness assessment, COVID19 related research, neural networks for prediction of purchase intention and application of machine learning in sentiment analysis. These articles are well suited for the selected theme, and they are pertinent to the aims and scope of the AIP journal.

Due to the pandemic, people have moved dramatically towards online platforms. E-commerce is one of the fields that show rapid growth. In order to maximise the potential, e-commerce readiness assessment is necessary. The article entitled E-Commerce Readiness Assessment in Sarawak selects Sarawak, a state of Malaysia, as a case study. The article proposes a research model that uses the perceived risk, perceived usefulness and perceived quality of products, and the intention of adoption. The validity of the proposed model is evaluated according to the reliability, construct validity, convergent validity, and discriminant validity. The findings obtained could give insight to other parts of the world. Beside e-commerce, education is also encountering a major shift. Trialability and Purposefulness: Their Role Towards Google Classroom Acceptance Following Educational Policy is an article that investigates the acceptance and adoption of Google Classroom as an online learning platform in the Philippines. The finding of the research showed that Google Classroom is an appropriate platform for tertiary education.

A notable article for the current pandemic crisis is Modelling COVID-19 Hotspot Using Bipartite Network Approach. The objective of this research is to formulate a bipartite network model of COVID19 transmissions that improves the conventional model and focuses on indirect transmission. The model incorporates patient mobility data to address the assumption of population homogeneity. The outcome of this research is suitable for forming a framework for public health authorities to identify the source of infection and high-risk people in the COVID-19 cases to control the transmission at the initial stage.

Two other articles, which utilise the machine learning approach to increase their competitiveness in the business domain, show the interdisciplinary nature of ICT. The article A Neural Network-Based Approach in Predicting Consumers' Intentions of Purchasing Insurance Policies uses a neural network technique to predict the propensity of consumers to purchase insurance policies. The proposed technique works by using the construction of features from historical data and feeding them to a neural network. The prediction task is carried out by the developed neural network model. In contrast, in the article Sentiment Analysis for Thai Language in Hotel Domain Using Machine Learning Algorithms, the proposed method uses a machine learning technique to perform a sentiment analysis of polarity classification in the area of hotel customer reviews in the Thai language.

Overall, the articles published in this special issue further emphasise the importance of information and communication technologies for digital inclusivity, especially in these challenging times. The contributions of the articles move us a small step in the right direction, which in time may add up to a giant leap forward.

Acta Informatica Pragensia is published by Prague University of Economics and Business, Czech Republic.

ISSN: 1805-4951 